\title{
FIGO Stage IIIC
}

National Cancer Institute

\section{Source}

National Cancer Institute. FlGO Stage IIIC. NCI Thesaurus. Code C96258.

A FIGO stage term that applies to gynecologic cancers. There is no FIGO stage IIIC for cervical cancer; for endometrial cancer, FIGO stage IIIC is subdivided into stages IIIC1 and IIIC2; in FIGO stage IIIC1, there is regional metastasis to pelvic lymph nodes; for FIGO stage IIIC2, there is regional metastasis to para-aortic lymph nodes with or without metastasis to pelvic lymph nodes. 